\title{
Obstructive sleep apnea in Treacher Collins syndrome
}

\author{
Harriet Akre $\cdot$ Britt Øverland · Pamela Åsten • \\ Nina Skogedal $\cdot$ Ketil Heimdal
}

Received: 16 March 2011 / Accepted: 11 May 2011/Published online: 29 May 2011

(c) The Author(s) 2011. This article is published with open access at Springerlink.com

\begin{abstract}
The aim of the present study was to investigate the prevalence of obstructive sleep apnea syndrome (OSAS) among the Norwegian population with Treacher Collins syndrome (TCS). A secondary aim was to establish whether TCS phenotype severity is associated with OSAS severity. A prospective case study design was used. Individuals who were 5 years old and above with a known diagnosis of TCS in Norway were invited to participate in a study. The study included genetic testing, medical and dental examinations and polysomnography. All participants demonstrated disturbed respiration during sleep; $18 / 19$ met the diagnostic criteria for OSAS. Subjectively evaluated snoring was not a reliable predictor of OSAS. We found no significant association between TCS phenotype severity and the severity of OSAS. OSAS is common in TCS, but there is no association with the phenotype severity. Individuals diagnosed with TCS must undergo sleep studies to identify the presence of OSAS.
\end{abstract}

Keywords Sleep apnea - Craniofacial syndromes · Phenotype $\cdot$ Snoring

H. Akre $(\bowtie) \cdot$ B. Øverland

Sleep Unit, Department of Otorhinolaryngology-Head and Neck Surgery, Lovisenberg Diakonale Hospital, 0440 Oslo, Norway e-mail: haak@lds.no

P. Åsten · N. Skogedal

TAKO-Centre, Resource Centre for Oral Health in Rare Medical Conditions, Lovisenberg Diakonale Hospital, Oslo, Norway

K. Heimdal

Department of Medical Genetics, Oslo University Hospital, Oslo, Norway

\section{Introduction}

Obstructive sleep apnea syndrome (OSAS) is a common disorder, affecting $2-4 \%$ of children and adults [1-3]. Narrowing of the upper airways is a primary risk factor for OSAS [4]. This syndrome is caused by partial or complete obstruction of the upper airways during sleep despite ongoing inspiratory effort. These respiratory events lead to episodes of oxygen desaturation and sleep disruption, which harm the individual's physical and mental health [57]. OSAS is common among patients with craniofacial deformities [8]. The development of upper airway skeletal and soft tissue components is influenced by genetic factors, and an intricate relationship between these components determines the ultimate shape, patency and function of the upper airways. The obstructions in craniofacial syndromes are located at multiple sites in the upper airways, often caused by hypoplasia of the maxilla and/or the mandible.

Treacher Collins Syndrome (TCS) is an autosomal dominant disorder of craniofacial development with an incidence of approximately 1 in 50,000 live births [9, 10]. Penetrance of the genetic mutations underlying TCS is high, yet inter- and intra-familial variation in the severity of the phenotype is a striking feature of the condition [11, 12]; $60 \%$ of cases are assumed to be de novo mutations [13, 14]. Features characterising TCS include bilateral downward slanting palpebral fissures, frequently accompanied by colobomas of the lower eyelid with a paucity of eyelashes medial to the defect; abnormalities of the external ear, atresia of the auditory canal, and bilateral conductive hearing loss; hypoplasia of the zygomatic complex and mandible; and cleft palate $[12,15]$. Although people with TCS are considered at high risk for developing OSAS [16] due to 
micrognathia, few case reports describe this association $[16,17]$.

The aim of the present study was to examine all individuals from 5 years of age with a known diagnosis of Treacher Collins syndrome (TCS) in Norway for OSAS. A secondary aim was to establish whether TCS phenotype severity is associated with OSAS severity. OSAS poses a health risk for these individuals, with potential effects on cognitive function, work and school performance, and, possibly, mortality.

\section{Methods}

\section{Patients}

An invitation to an extended medical examination was sent to all patients aged 5 and above in October 2008 registered at the TAKO centre (a national resource centre for oral health in rare medical conditions), Lovisenberg Diakonale Hospital, Department of Medical Genetics and Centre for Rare Disorders, Oslo University Hospital, Rikshospitalet and seven centres in the Norwegian Resource System for persons with deaf blindness. Requests were also addressed to the Departments of Medical Genetics at all university hospitals in Norway and the support group Norwegian Craniofacial Association.

This resulted in 36 eligible subjects with TCS who were invited to attend and 23 accepted to participate. The Regional Committee for Medical Research Ethics evaluated and recommended the study protocol. All the 23 participants gave an informed consent. Below 16 years of age, informed consent was obtained by the child and their parents.

\section{Medical and oral examination}

A clinical geneticist saw all patients for diagnostic purposes. Patients from families with unknown mutation status were offered genetic testing as part of the routine clinical work-up. Genetic testing (Sanger sequencing) was performed by a clinical genetic laboratory. Eligible participants not thought to fulfil a clinical diagnosis of TCS and in whom genetic testing was not performed (or negative) were excluded from the study.

In addition, participants underwent a full ear nose and throat examination including endoscopic evaluation of the upper airway from the same experienced otorhinolaryngologist. They underwent a hearing test by a certified audiologist. They were all asked specific questions about symptoms of possible sleep apnoea syndrome. They spent the night in hospital for a polysomnography (PSG) evaluation. Body mass index was calculated from weight and height $\left(\mathrm{kg} / \mathrm{m}^{2}\right)$.
This collaborative study also included examinations of intraoral conditions and salivary secretion by a specialist in paediatric dentistry. Dental casts were made and lateral cephalogram and orthopantomogram taken. All underwent ultrasound examinations of the major salivary glands. A speech and language pathologist assessed orofacial functions. Participants from school age answered a package of quality of life-questionnaires.

\section{Phenotypic expression}

The patients were classified according to a system developed by Teber et al. in 2004 [18], as a method for quantifying phenotypic expression in TCS patients. The main clinical features (downward slanting palpebral fissures, lower eyelid coloboma, hypoplasia of zygomatic complex, hypoplasia of mandible, microtia) scored two points each. Accessory features (cleft palate, atresia of external ear canal, conductive deafness, tracheostoma, choanal stenosis/ atresia, preauricular tags, delayed motor development, delayed speech development) scored one point each. The geneticist and otorhinolaryngologist registered all features, except delayed motor and speech development, at clinical examinations. Information of development was collected by anamnestic interviews. Conductive deafness was defined as hearing loss over $25 \mathrm{~dB}$. The overall facial phenotype was classified as mild (scored with one point) or severe (scored with two points) based on photographs viewed by all collaborators.

The maximum sum of scores is 20 points if all relevant data are available. Patients with a score of $0-10 / 20$ are regarded as mildly affected and patients with scores 11-20/ 20 are regarded as severely affected. If available data are incomplete, the maximum score will be less than 20 .

\section{Hearing tests}

Hearing loss was measured using pure-tone audiometry, according to recommended procedures (ISO 8253-1 1989). A Madsen Auricle audiometer calibrated according to ISO standards (ISO 389-1 1998, ISO 389-3 1994) was used. The test was carried out in a quiet room. Air conduction thresholds were obtained separately for the left and right ear. The frequencies 500, 1,000, 2,000, 4,000 Hz, WHO (World Health Organization) M4, were used to estimate mean hearing loss.

\section{Polysomnography}

All patients underwent attended overnight Polysomnography (PSG) (Embla, Resmed, Norway) at the Sleep Laboratory, Lovisenberg Diakonale Hospital, Oslo, Norway. In the children, there were also performed simultaneously 
video and audio recordings. The PSG recordings included a four-channel electroencephalogram (EEG) (C3/A2, C4/A1, O2/A1, O4/A2), right and left electrooculogram (EOG), and submental electromyogram (EMG). Ribcage and abdominal wall movements were measured using respiratory inductance plethysmography. Flow was measured with nasal pressure transducer and the arterial oxygen saturation was monitored via pulse oximetry. Electrocardiogram (ECG), body position and EMG from both legs were also included.

Sleep was scored for sleep stages according to the guidelines from Rechtschaffen and Kales [19]. Arousals were defined according to American Sleep Disorders Association criteria [20]. In the adults, apnea was defined as a more than $90 \%$ reduction of flow for more than $10 \mathrm{~s}$. They were classified as obstructive in the presence of ribcage and abdominal wall movements and central with absent respiratory effort. Hypopneas were defined as a more than $50 \%$ decrease in flow for more than $10 \mathrm{~s}$ with subsequent oxygen desaturation of at least $3 \%$ or an arousal. In the children, an obstructive apnea was defined as at least two breaths with more than $90 \%$ reduction in flow in the presence of ribcage and abdominal movement. A central apnea was defined as at least two breaths with more than $90 \%$ reduction without absent respiratory effort with subsequent oxygen desaturation of at least $3 \%$ or an arousal, or if the event lasts for more than $20 \mathrm{~s}$. Hypopneas were defined as a more than $50 \%$ decrease in flow for at least two breaths with subsequent oxygen desaturation of at least $3 \%$ or an arousal [21]. The hypopneas were not classified further for either the adults or the children.

Apnea hypopnea index (AHI) was defined as the number of apneas and hypopneas per hour of sleep, while oxygen desaturation index (ODI) was defined as the number of oxygen desaturations $\geq 3 \%$ per hour of sleep. Mild obstructive sleep apnea (OSA) was defined as AHI 5-15 in adults and AHI 1-5 in children, moderate OSA as AHI 15-30 in adults and AHI 5-10 in children and severe OSA as $\mathrm{AHI}>30$ in adults $\mathrm{AHI}>10$ in children.

For estimating snoring, we used a VAS-scale, where the participants were asked to assess how often they snore. The parents filled in the score for children younger than 18 years of age.

\section{Statistical analysis}

The analysis was performed using SPSS 18.0 for Windows (SPSS INC., Chicago, IL, USA). Descriptive analysis was used to examine and present the data. The Pearson $\chi^{2}$ was used to test if there were differences in the clinical phenotype features between the severity groups of OSA. The level of significance was established at $5 \%$.

\section{Results}

As a result of clinical and genetic examinations, two participants were excluded from the analyses due to unconfirmed TCS diagnosis. A further two individuals were excluded due to the presence of an additional neuromuscular condition, which could influence orofacial function and breathing. The study group thus consisted of 19 individuals between the ages 5 and 74 from 14 families. Participants between 5 and 18 years of age were classed as children in the analysis. Table 1 defines the basic characteristics of the sample. Based on BMI, the majority of participants were of normal weight or below.

Results of genetic testing were available for 17/19 participants (13/14 families). The causative mutation was detected in all but three families (mutation detection rate $10 / 13=77 \%$ ). No mutations were found in more than one family. Mutations were predicted to lead to loss of function of the gene in all families. The sample was too small to permit genotype-phenotype analyses.

Table 2 describes the participants' phenotypic characteristics. Among our group the most common features were downward slanting palpebral fissures (15/19), hypoplasia of the zygomatic complex (16/19), hypoplasia of the mandible (17/19), conductive hearing loss (17/19) and atresia of the external ear canal (11/19). Cleft palate was present in five cases. Two of the adults have developed unilateral facial palsy in association with corrective surgery. Based on the sum of scores from clinical findings, 13 individuals were classified as severely affected and 6 as mildly affected.

The PSG demonstrated that all participants experienced breathing disturbances during sleep. The mean AHI (SD) was 30 (21.5) for adults and 5 (4.3) for children. Table 3 describes the distribution of severity of OSA among children and adults. All the adults, and all but one of the children, met the diagnostic criteria for OSA. In this child, we observed snoring and flattening of the flow curve, indicating increased resistance in the upper airway. $72.8 \%$ of the adults and $37.5 \%$ of the children presented with moderate to severe OSA. In both adults and children, the

Table 1 Basic characteristics of the participants $(N=19)$

\begin{tabular}{llllll}
\hline & \multicolumn{2}{l}{ Children $(n=8)$} & & \multicolumn{2}{l}{ Adults $(n=11)$} \\
\cline { 2 - 3 } & Mean & SD & & Mean & SD \\
\hline Age & 11.0 & 4.7 & & 47.2 & 13.2 \\
BMI & 17.7 & 4.6 & & 25.9 & 5.0 \\
Gender & Female & Male & & Female & Male \\
& 6 & 2 & & 7 & 4 \\
\hline
\end{tabular}




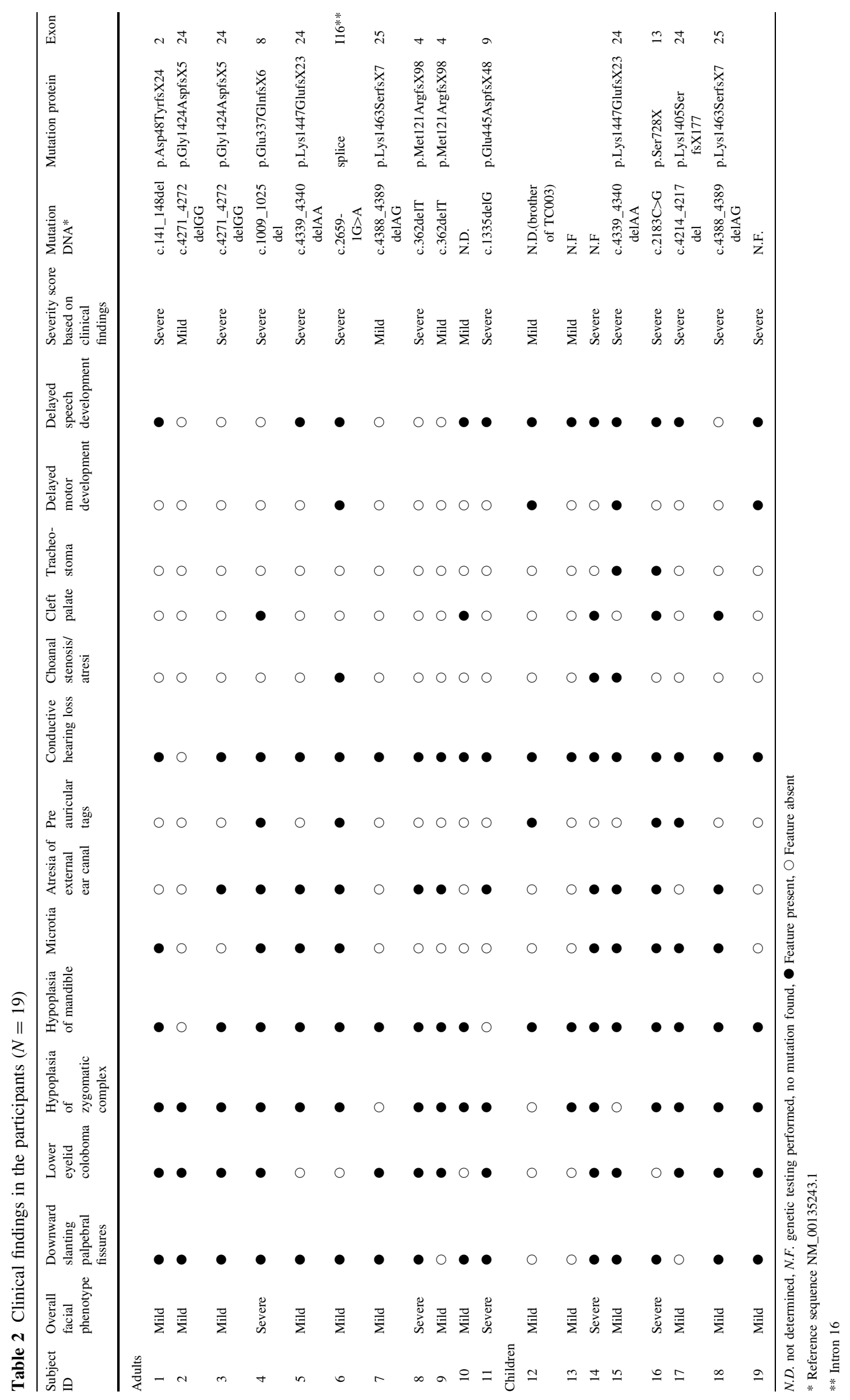


Table 3 Distribution of AHI grouped by severity of OSA in children and adults

\begin{tabular}{llllll}
\hline & \multicolumn{2}{l}{ Children $(n=8)$} & & \multicolumn{2}{c}{ Adults $(n=11)$} \\
\cline { 2 - 3 } & $n$ & & & $n$ & $\%$ \\
\hline None & 1 & 12.5 & & 0 & 0.0 \\
Mild OSAS & 4 & 50.0 & & 3 & 27.3 \\
Moderate OSAS & 2 & 25.0 & & 4 & 36.4 \\
Severe OSAS & 1 & 12.5 & & 4 & 36.4 \\
\hline
\end{tabular}

Table 4 Respiratory disturbances recorded by PSG, the first 11 are adults, the last 8 are children

\begin{tabular}{lrrllc}
\hline ID & AHI & ODI & $\begin{array}{l}\text { Lowest } \\
\text { SAO2 }\end{array}$ & $\begin{array}{l}\text { Average } \\
\text { SaO2 }\end{array}$ & $\begin{array}{l}\text { Subjective } \\
\text { snoring }\end{array}$ \\
\hline 1 & 21.7 & 17.0 & 88 & 94.5 & 5.1 \\
2 & 7.8 & 8.5 & 89 & 94.9 & 7.8 \\
3 & 38.6 & 21.7 & 89 & 95.8 & 4.8 \\
4 & 32.5 & 20.0 & 83 & 95.0 & 6.9 \\
5 & 75.0 & 61.6 & 82 & 95.7 & 10.0 \\
6 & 63.7 & 57.2 & 81 & 94.7 & 10.0 \\
7 & 21.6 & 19.9 & 89 & 94.7 & 10.0 \\
8 & 25.1 & 22.8 & 87 & 95.5 & 10.0 \\
9 & 18.1 & 10.3 & 85 & 93.7 & 5.6 \\
10 & 12.1 & 24.1 & 80 & 92.6 & 9.4 \\
11 & 14.0 & 13.2 & 81 & 96.2 & 10.0 \\
12 & 5.1 & 5.5 & 89 & 96.6 & 7.3 \\
13 & 1.5 & 0.9 & 94 & 97.2 & 5.5 \\
14 & 8.4 & 6.9 & 92 & 97.7 & 9.0 \\
15 & 7.7 & 3.0 & 93 & 98.0 & 3.0 \\
16 & 12.7 & 15.8 & 80 & 96.4 & 0.0 \\
17 & 1.7 & 1.5 & 94 & 97.5 & 7.8 \\
18 & 1.8 & 6.2 & 91 & 96.8 & 8.0 \\
19 & 0.7 & 0.2 & 91 & 98.2 & 3.0 \\
\hline & & & & &
\end{tabular}

majority of the respiratory events were obstructive. AHI varied greatly between participants. Deeper oxygen desaturations were observed in the adults than in the children.

All the adults scored 4.8 or more on the VAS-scale for subjective snoring. The children had variable distribution of registrations along the VAS-scale (0.0-9.0). We found no correlation between AHI and subjectively evaluated snoring. Table 4 shows the respiratory events and oxygen saturation statistics together with each participant's score for subjective snoring.

We did not find a significant difference in AHI according to the severity of phenotype by clinical findings, but there was a tendency for higher AHI among participants with the severe phenotype (Fig. 1). The contributions of each of the single features were tested, and neither of these was significantly related to OSA.

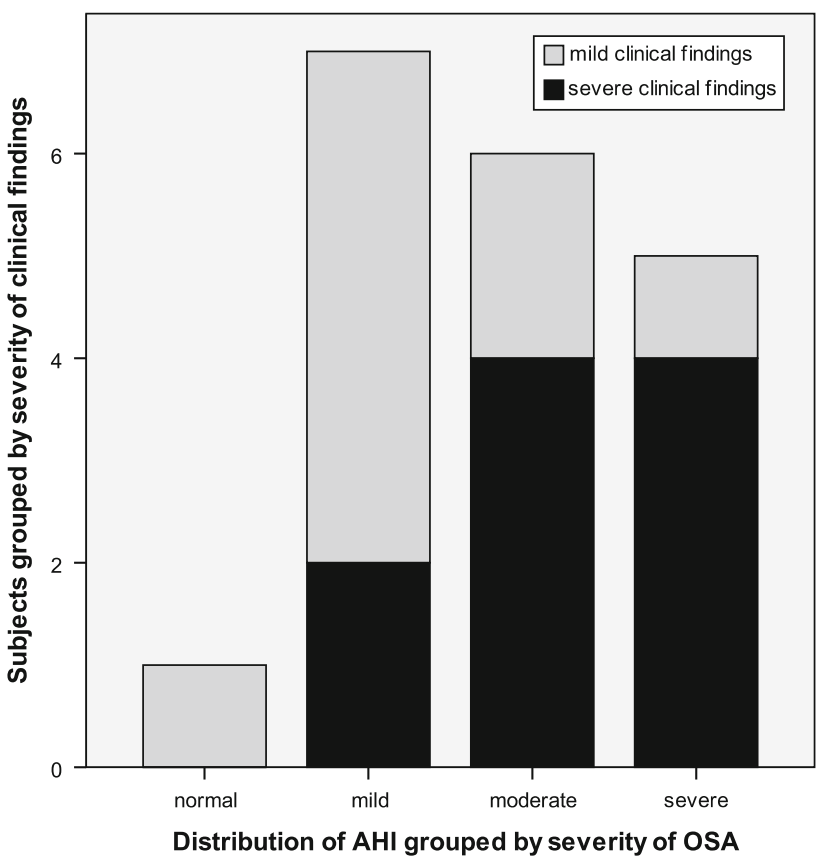

Fig. 1 Distribution of AHI grouped by severity of OSA and separated according to severity of clinical findings

\section{Discussion}

The increased incidence of OSAS in patients with craniofacial deformities is well-known [22, 23], but there are only a few case reports considering OSAS in TCS. All but one of our study participants had OSAS. Diagnosis and treatment is important as OSAS poses serious health risks, including cardiovascular disease and impaired cognitive development [24-26].

During the last 50 years, there have been approximately $50,000-60,000$ births per year in Norway. Assuming an annual incidence rate of $1: 50,000$ and some perinatal mortality, there may have been $60-80$ TCS cases in Norway during this period. We acknowledge that some individuals with mild phenotype may be unidentified, but believe that all the individuals currently diagnosed with TCS in Norway were invited to participate in the study.

Our case series has a severity score comparable with that of Teber et al.: 13/19 (68\%) severe cases in this case series compared with 26/35 (74\%) in Teber et al. We do not know if there are differences between the sample investigated and the individuals who chose not to participate. Interestingly, some of those who did not participate were later diagnosed with obstructive sleep apnea. In conclusion, we believe the sample investigated is representative of the Norwegian TCS population and typical of a population presenting to the health service with this diagnosis.

Based on clinical findings applied to the system developed by Teber et al. 13 (68\%) participants were classified 
with a severe phenotype of TCS. There was no significant difference in AHI between the severe and mild phenotype, but this could be due to the small number of subjects in our study group. The use of standard clinical scoring systems across studies might permit meta-analyses to clarify this question in the future.

Not all of the specific features of TCS are likely to cause OSA. Hypoplasia of the mandible and zygomatic complex, cleft palate and choanal atresia are known contributors to narrowing of the airway. We therefore tested each of these specific features against severity groups of OSA. We did not find any significant correlation, but again the low number of participants probably causes this. Delayed speech development is seen in many of the patients. This may be caused by hearing loss, but also OSA can contribute to delay of speech because of cognitive impairment due to sleep disruption and oxygen desaturation [27].

The only participant who did not have OSAS, was classified clinically as having the severe phenotype (19, score 12/20). This demonstrates that clinical examination and severity of the phenotype are not sufficient to exclude OSAS. Subjectively evaluated snoring in people with TCS was also not a reliable clinical indicator of OSAS. A PSG must be carried out in order to confirm the presence or absence of OSAS. In children with TCS, it is advisable that the PSG is repeated throughout childhood since the size of the airways and the soft tissue (adenoids and tonsils) alters as the child develops. Some children with OSAS may benefit from treatment with continuous positive airway pressure (CPAP) until suitable conditions for corrective surgery are obtained [28]. Case reports have indicated that corrective surgery of the mandible is an effective treatment for OSAS [16]. Although most of the adults in our group had undergone corrective surgery to improve respiration, they continued to experience moderate to severe OSAS. This finding demonstrates the importance of post-surgical retesting in order to identify persisting sleep apnea.

\section{Conclusion}

All patients in this study presented with breathing disturbances during sleep. Only one had been diagnosed prior to the study. This indicates a high prevalence of obstructive sleep apnea syndrome in TCS and highlights the value of offering diagnostic sleep studies to all individuals diagnosed with TCS. The severity of TCS phenotype could not be used to estimate the degree of OSA. The timing and frequency of sleep studies should be evaluated on an individual basis.

Conflict of interest None of the authors has any financial or other relationship that might lead to a conflict of interest.
Open Access This article is distributed under the terms of the Creative Commons Attribution Noncommercial License which permits any noncommercial use, distribution, and reproduction in any medium, provided the original author(s) and source are credited.

\section{References}

1. Gislason T, Benediktsdottir B (1995) Snoring, apneic episodes, and nocturnal hypoxemia among children 6 months to 6 years old. An epidemiologic study of lower limit of prevalence. Chest 107(4):963-966

2. Redline S, Tishler PV, Schluchter M, Aylor J, Clark K, Graham G (1999) Risk factors for sleep-disordered breathing in children. Associations with obesity, race, and respiratory problems. Am J Respir Crit Care Med 159(5 Pt 1):1527-1532

3. Young T, Palta M, Dempsey J, Skatrud J, Weber S, Badr S (1993) The occurrence of sleep-disordered breathing among middle-aged adults. N Engl J Med 328(17):1230-1235

4. Sher AE (1990) Obstructive sleep apnea syndrome: a complex disorder of the upper airway. Otolaryngol Clin North Am 23(4):593-608

5. Budhiraja R, Budhiraja P, Quan SF (2010) Sleep-disordered breathing and cardiovascular disorders. Respir Care 55(10): 1322-1332

6. Tsai JC (2010) Neurological and neurobehavioral sequelae of obstructive sleep apnea. Neuro Rehabil 26(1):85-94

7. Zizi F, Jean-Louis G, Brown CD, Ogedegbe G, Boutin-Foster C, McFarlane SI (2010) Sleep duration and the risk of diabetes mellitus: epidemiologic evidence and pathophysiologic insights. Curr Diab Rep 10(1):43-47

8. Hoeve LJ, Pijpers M, Joosten KF (2003) OSAS in craniofacial syndromes: an unsolved problem. Int J Pediatr Otorhinolaryngol 67(Suppl 1):S111-S113

9. Fazen LE, Elmore J, Nadler HL (1967) Mandibulo-facial dysostosis. (Treacher-Collins syndrome). Am J Dis Child 113(4):405-410

10. Rovin S, Dachi SF, Borenstein DB, Cotter WB (1964) Mandibulofacial dusostosis, a familial study of five generations. J Pediatr 65:215-221

11. Dixon MJ, Marres HA, Edwards SJ, Dixon J, Cremers CW (1994) Treacher Collins syndrome: correlation between clinical and genetic linkage studies. Clin Dysmorphol 3(2):96-103

12. Marres HA, Cremers CW, Dixon MJ, Huygen PL, Joosten FB (1995) The Treacher Collins syndrome. A clinical, radiological, and genetic linkage study on two pedigrees. Arch Otolaryngol Head Neck Surg 121(5):509-514

13. Jones KL, Smith DW, Harvey MA, Hall BD, Quan L (1975) Older paternal age and fresh gene mutation: data on additional disorders. J Pediatr 86(1):84-88

14. Marszalek B, Wojcicki P, Kobus K, Trzeciak WH (2002) Clinical features, treatment and genetic background of Treacher Collins syndrome. J Appl Genet 43(2):223-233

15. Dixon MJ (1995) Treacher Collins syndrome. J Med Genet 32(10):806-808

16. Johnston C, Taussig LM, Koopmann C, Smith P, Bjelland J (1981) Obstructive sleep apnea in Treacher-Collins syndrome. Cleft Palate J 18(1):39-44

17. Ueda K, Tajima S, Tanaka Y, Imai K, Byun S, Byun M (1996) Correction of severe sleep apnea in a case of Treacher-Collins syndrome. Eur J Plast Surg 19:320-322

18. Teber OA, Gillessen-Kaesbach G, Fischer S, Bohringer S, Albrecht B, Albert A et al (2004) Genotyping in 46 patients with tentative diagnosis of Treacher Collins syndrome revealed 
unexpected phenotypic variation. Eur J Hum Genet 12(11):879-890

19. Rectscaffen A, Kales A (1968) A manual of standardized terminology, techniques and scoring system for sleep stages of human subjects. UCLA Brain Information Service/Brain Research Institute, Los Angeles

20. ASDA EEG arousals (1992) Scoring rules and examples: a preliminary report from the Sleep Disorders Atlas Task Force of the American Sleep Disorders Association. Sleep 15(2):173-184

21. Iber C, Ancoli-Israel S, Chesson A, Quan S (2007) The AASM Manual for the scoring of sleep and associated events. Rules, terminology and technical specifications

22. Brooks LJ (2000) Genetic syndromes affecting breathing during sleep. In: Loughlin GM, Carroll JL, Marcus CL (eds) Sleep and breathing in children. Marcel Dekker Inc, New York-Basel, pp 737-754

23. Ferraro NF (2000) Craniofacial development and the airway during sleep. In: Loughlin GM, Carroll JL, Marcus CL (eds)
Sleep and breathing in children. Marcel Dekker, New YorkBasel, pp 293-312

24. Kwok KL, Ng DK, Chan CH (2008) Cardiovascular changes in children with snoring and obstructive sleep apnoea. Ann Acad Med Singapore 37(8):715-721

25. Kheirandish-Gozal L, Bhattacharjee R, Gozal D (2010) Autonomic alterations and endothelial dysfunction in pediatric obstructive sleep apnea. Sleep Med 11(7):714-720

26. Kheirandish L, Gozal D (2006) Neurocognitive dysfunction in children with sleep disorders. Dev Sci 9(4):388-399

27. Canessa N, Castronovo V, Cappa SF, Aloia MS, Marelli S, Falini A, et al. (2011) Obstructive Sleep Apnea: brain structural changes and neurocognitive function before and after treatment. Am J Respir Crit Care Med 183(10):1419-1426

28. Miller SD, Glynn SF, Kiely JL, McNicholas WT (2010) The role of nasal CPAP in obstructive sleep apnoea syndrome due to mandibular hypoplasia. Respirology 15(2):377-379 